\title{
SPHERE SHELL SPACE 3D GRID
}

\author{
Gang WAN, Xuefeng CAO*, Feng LI, Ke LI,
}

\author{
Zhengzhou Institute of Surveying and Mapping, 450052 Zhengzhou, Henan, China
}

KEY WORDS: sphere shell, global spatial grid, discrete global grid, spheroid spatial grid, grid subdivision, grid coding

\begin{abstract}
:
The global spatial grid is an important spatial data model for Digital Earth. Discrete Global Grid provides a means of rasterizing the earth surface, and then has been considered as the basic data model for digital globes. But Discrete Global Grid is only confined to the surface, because it can not reach to the earth inside and outside. The Sphere Shell Space 3D Grid (SSSG) proposed here provides a new spatial reference framework for the whole earth 3D space for Digital Earth. The basic conceptions and requirements of SSSG have been discussed in details. It is designed to represent spatial objects distributed from underground, land, sea, air to space within a uniform framework, and also to uniformly organize various kinds of spatial information in each earth sphere shells.
\end{abstract}

\section{INTRODUCTION}

The range of human activities is more and more wide, and then global issues have been paid higher degree of concern. The research about global space is more in-depth. Various global observation system and plans have been put forward in geology, oceanography, climatology, geography, earth system. It is obvious that global 3D distributions, spanning multi-temporal and spatial scales, cross specialties are new features of human activities. This trend has provided new demands for global spatial grid, global geographic information system and digital earth.

Discrete Global Grid gives a feasible way to meet this trend, and it has been widely used as the basic data model of digital earth (Goodchild, 2000). There have been a lot of researches on Discrete Global Grid data model, such as global data multihierarchical structure and indexing (Ottosm, 2002), global-scale image archiving model (Seong, 2005), and so on. The base of Discrete Global Grid model is grid subdivision. Usually, grid of GDG data model are mainly based on normal-polyhedron-based sphere partition, such as octahedron-based sphere grid (Dutton, 1999, Cui, 2009), dodecahedron-based sphere grid (Wickman, 1974), icosahedron-based sphere grid (Fekete, 1990).

In recent years, several global 3D grid models have been proposed, such as Spheroid Latitude-Longitude Grid, Spheroid Yin-Yang Grid (Kageyama, 2004), Cubed-Sphere Grid (Tsuboi, 2008), Adaptive-Mesh Refinement Grid (Stadler, 2010), Sphere Degenerated Octree Grid (YU Jieqing, 2009). These 3D grid models are mainly used in earth system simulation, including geodynamo and mantle convection, global climate change, etc. But there are two problems. First, the partition method of the surface grid and the spheroid grid is dissevered. Global surface grid focus on the spatial data of earth surface, whereas the data inside and outside of earth is ignored. In Global 3D grid models, the earth is considered as a sphere, and the partition is took place in the 3D space. Then it is naturally to organize 3D data.

\section{GLOBAL SPATIAL GRID}

Global spatial grid consists of sphere grid and spheroid grid according to the division target. The main content of global spatial grid includes grid subdivision, grid coding, grid distortion, grid index and grid analysis.

\subsection{Sphere Grid}

In recent years, the development of sphere grid is progressive in stages. There are various spherical subdivision and coding models. They could be classified into three categories by the model principle.

2.1.1 Discrete Global Grid: A discrete global grid is defined as a partitioning of the surface of the globe into approximately identical tiles (Goodchild, 2000). Various discrete global grid models are different at polyhedron, map projection, coding. There are five known Platonic solids (Finger 1): the tetrahedron, the cube, the octahedron, the dodecahedron and the icosahedron (Sahr, 2003). And the optional map projection includes Lambert Projection, Gnomonic Projection, Fuller-Gray Projection, Snyder Equal-Area Projection, Equal Angle Ratio Projection, etc.

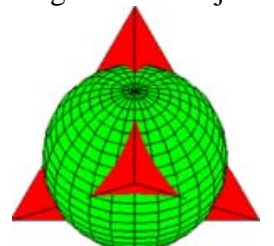

Tetrahedron

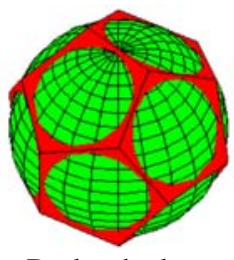

Dodecahedron

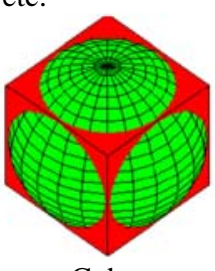

Cube

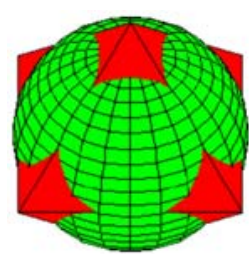

Octahedron
Finger 1. Five Platonic Polyhedron

2.1.2 Spherical Lon-Lat Grid: A spherical uses the latitude line and longitude line to partition the globe into tiles. The longitude and latitude interval could be equal or unequal. Equal interval Lon-Lat grid has been used in Google Earth, Virtual Earth, World Wind, etc. Unequal Lon-Lat grid includes ellipsoid quad tree grid (Ottosm, 2002), DTED grid, degenerated quadtree grid (SDQ).

\footnotetext{
* Corresponding author: Xuefeng CAO, cxfchxy@163.com.
} 


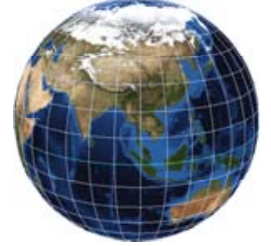

(a) Equal Spacing Lon-Lat Grid

Finger 2. Spherical Lon-Lat Grid

2.1.3 Spherical Voronoi Grid: Lukatela proposed a spherical voronoi grid which is used as a seamless global terrain model in the hipparchus system. Kolar provided a similar model, but with multi-level structure (Kolar, 2004). Spherical voronoi grid is more agile, but the hierarchy structure is defined explicitly, which is not feasible for global multi-scale model construction and operation.

2.1.4 Spherical Equal Portion Grid: Different from other spherical grid models, spherical equal portion grid divide the globe into several equal faces firstly, and then the subdivision operations take place in each face. In the HEALPix grid (Gorski, 2005), the globe is divided into 12 equal area faces, and each face is recursively partitioned into 4 subcells. In GeoFusion grid, the globe is divided into 6 approximate equal area faces, and each face is recursively subdivided into 4 subcells.

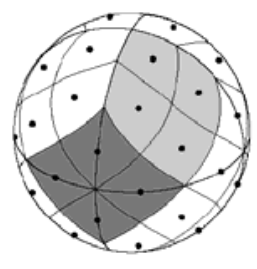

(a) HEALPix Grid

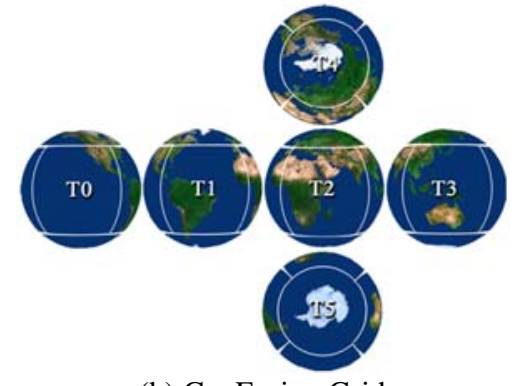

(b) GeoFusion Grid
Finger 3. Spherical Equal Portion Grid

\subsection{Spheroid Grid}

There are less spatial subdivision and coding models in spheroid grid than spherical grid. As we known, spheroid grid includes Spheroid Latitude-Longitude Grid, Spheroid Yin-Yang Grid (Kageyama, 2004), Cubed-Sphere Grid (Tsuboi, 2008), Adaptive-Mesh Refinement Grid (Stadler, 2010), Sphere Degenerated Octree Grid (YU Jieqing, 2009).

These 3D grid models are mainly used in earth system simulation, including geodynamo and mantle convection, global climate change, etc. Spheroid grid takes virtual spheroid as the division target. A virtual spheroid is a normal spheroid with a variable radius.

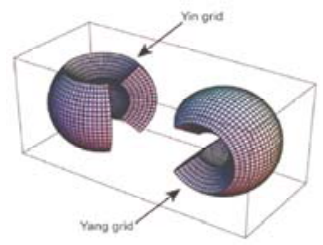

(a) Yin-Yang Grid

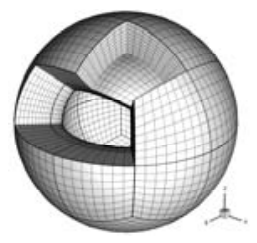

(b) Cubed-Sphere Grid

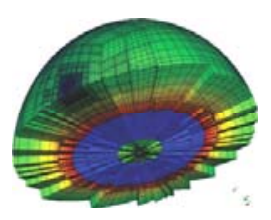

(c) Adaptive-Mesh Refinement Grid

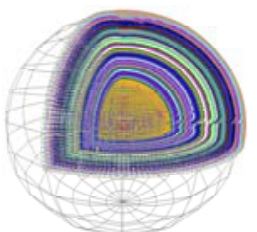

(d) Sphere Degenerated Octree Grid
Finger 4. Spheroid Grid

\section{ANALYSIS AND REQUIREMENTS}

\subsection{Analysis}

There are two problems in current global spatial grid research. First, the partition method of the spherical grid and the spheroid grid is dissevered. The spherical grid focus on the spatial data of earth surface, whereas the data inside and outside of earth is ignored. In the spheroid grid models, the partition of the earth is took place in the $3 \mathrm{D}$ space. Then it is naturally to organize 3D data with solid grid cells. But the surface data, such as remote sensing images, elevation data, could not be processed favourably.

Second, the sphere shell 3D structure, which is the basic spatial character of earth system, has not been reflected distinctly. In fact, the spatial information granularity and the modelling and representation requirement of different level of space are different.

\subsection{Requirements}

We present 10 requirements for the subdivision and coding model construction of Global Spatial 3D Grid.

1. Sphere shell structure: Sphere shell structure feature is the basic characteristic of earth system. The information granularity is different between different sphere shells, and it is the same for spatial data observation and modelling.

2. Cover completeness: Continuity, seamless, no overlap of grid cells are basic feature of global grid. It is also the requirement of SSSG.

3. Subdivision hierarchy: This is the basic feature of global grid.

4. Coding unique: It is also the unique characteristic of global grid.

5. Multi-resolution: The ability of multi-resolution spatial data organization is based on the multi-resolution structure of the grid model.

6. Space certainty: Each grid cell has the unique certain position, area and volume.

7. Geometry stability: The changes of edge length, the area, the thickness, the volume of grid cell must be stable or convergent all over the whole grid system. Other else it is not useable in practice.

8. Framework adaptability: Different domains need different parameters of grid. So it is feasible to provide the adaptability of grid construction.

9. Geographic coherence: Geographic coordinates is the base of almost all of spatial data and relative algorithms. So that it is important to hold geographic coherence.

10. Interoperability: There are various grid models. If each grid is absolutely independent, it is hard to exchange data between different grids. The interoperability between different grid models is important for data integration. 


\section{SPHERE SHELL SPACE 3D GRID}

Sphere shell space 3D grid is designed based on the sphere shell structure of the earth. The whole 3D space of the earth system is considered as several homocentric sphere shells.

\subsection{Basic Concepts}

The sphere shell is the basic notion in this paper. It is different from earth system spheres, such as hydrosphere, biosphere and lithosphere. Sphere shell is the mathematic abstraction of earth spheres, which is defined in mathematic formula. It consists of the inside sphere shell surface, the outside sphere shell surface and the sphere shell volume.

The sphere shell surface is one sphere with a radius, which is denoted as $S_{r}$. The radius of inside sphere shell surface is $r$, and $r+\Delta r$ for the outside sphere shell surface.

The datum sphere shell surface is set up according to earth system spheres parameters. The normal sphere surface whose space includes the solid earth is called the starting sphere shell surface, which is a datum sphere shell surface also. The radius of the starting sphere shell surface is mean radius of earth.

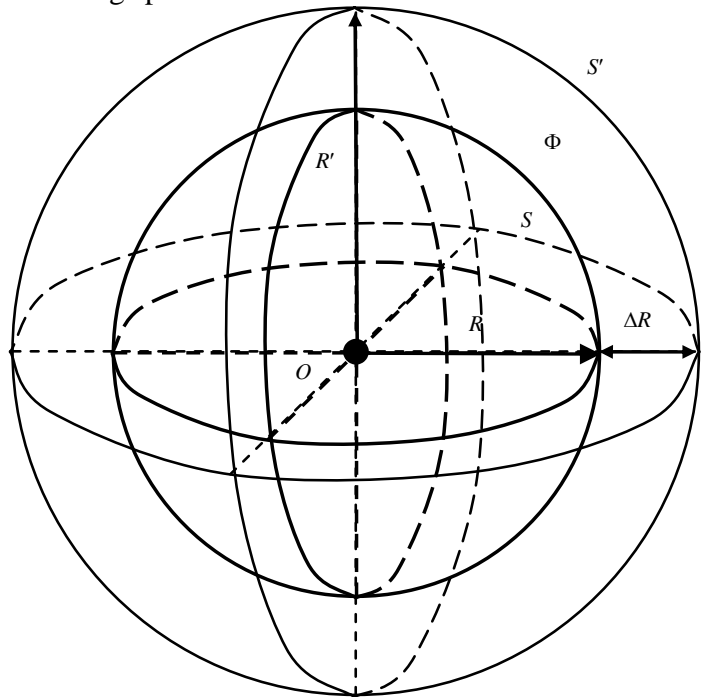

Figure 5. Sphere shell space geometric structure.

The sphere shell volume is the 3D space between inside and outside sphere shell surface; its radial thickness is $\Delta r$. The most inner sphere shell volume is a spheroid, not a shell, and its inner sphere shell surface radius is 0 , its thickness is $r$, then it is denoted as $\Phi_{r}$. All other sphere shell volumes are denoted as $\Phi_{\Delta r}$.

In the process of subdivision, the sphere shell surface and the sphere shell radial is partitioned into subcells, then the sphere shell volume is divided into solid subcells. So, the grid cells on sphere shell surface is called sphere shell surface grid cell, these subcells of the sphere shell radial are called sphere shell radial grid cell, and also the sphere shell volume grid cell. The formalization description of sphere shell volume grid cell is $\mathbf{V}=\{\mathbf{S}, \mathbf{E}, \mathbf{P}\}$. V denotes the sphere shell volume grid cell, $\mathrm{S}$ denotes the surfaces of sphere shell volume grid cell, E denotes the edges of sphere shell volume grid cell, $\mathrm{P}$ denotes the points of sphere shell volume grid cell.

\subsection{Design}

The process of partitioning and coding of $3 \mathrm{D}$ grid cells consists of two main steps: the partition of sphere shells and the subdivision of sphere shell volume.
The radius of the earth sphere is denoted as $R_{e}$. There are three datum sphere shell surfaces, their radius are $R_{e}, 7 / 6 R_{e}, 8 R_{e}$, corresponding to $\Phi_{R e} 、 \Phi_{R s} 、 \Phi_{R 8}$.

After the division of datum sphere shells, the sphere shell volume subdivision is carried out. First, one sphere shell volume is divided into several basic cells, called initial cells. Then these initial cells are partitioned according to the extended octree subdivision model. At last, the whole 3D grid cells compose the earth system space.

In fact, the whole process consist three stages: the partition of sphere shells, the initial partition of one sphere shell and the subdivision of sphere shell initial cells. All these three stages can be adjusted separately according to different domains.

\subsection{The Subdivision of Sphere Shell Surface}

The subdivision of sphere shell volume is taken place both at sphere shell surface and sphere shell radius, which is discussed as below.

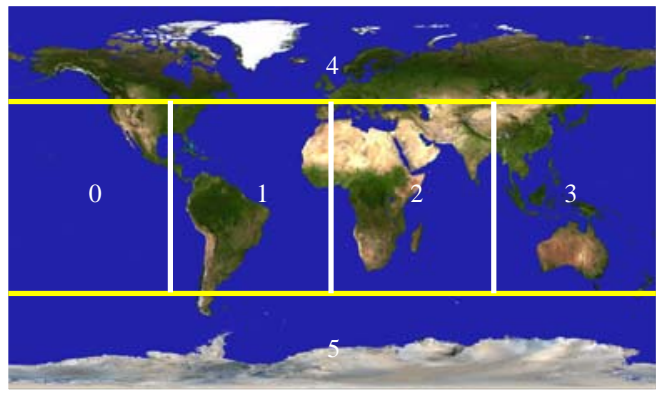

Figure 6. Six equal area faces

The subdivision of sphere shell surface is based on partition curve. First, the sphere shell surface is considered as a normal sphere, it is divided into six approximately equal area faces, four faces along low latitude and two faces near north and south polar, the boundary (yellow line in Finger 6) is latitude $\pm 45^{\circ}$. Second, the partition curve is constructed for each face. In Finger 7 and 8, the red lines are called $b$-curve, the green line is called $l$-curve, and three times subdivision of face 0 is shown.
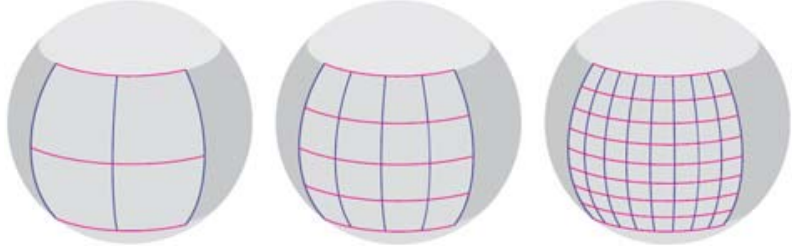

Figure 7. Sphere shell surface subdivision of face 0
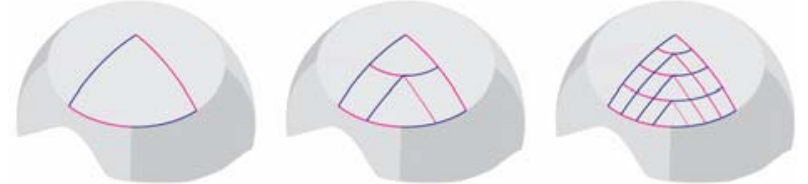

Figure 8. Sphere shell surface subdivision of face 4 The partition curve is extended from sphere surface to spheroid 3D space. In Finger 9 and 10, the gray fan is the partition curve surfaces.

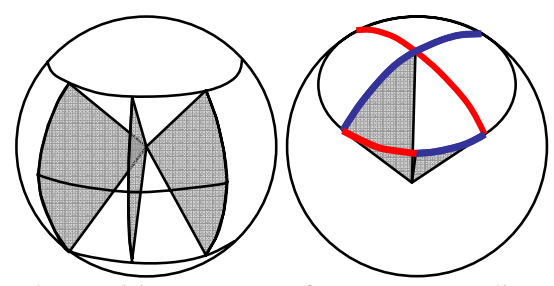

Figure 9. The partition curve surface corresponding to $l$-curve 

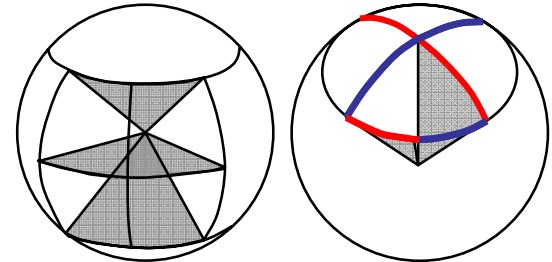

Figure 10. The partition curve surface corresponding to $b$-curve The subdivision of sphere shell radial is directly halving the thickness recursively.

\subsection{The Subdivision of Sphere Shell Volume}

Until now, global 3D space is divided into several sphere shells, and each sphere shell is divided into six basic sphere shell volume cells (Finger 11).
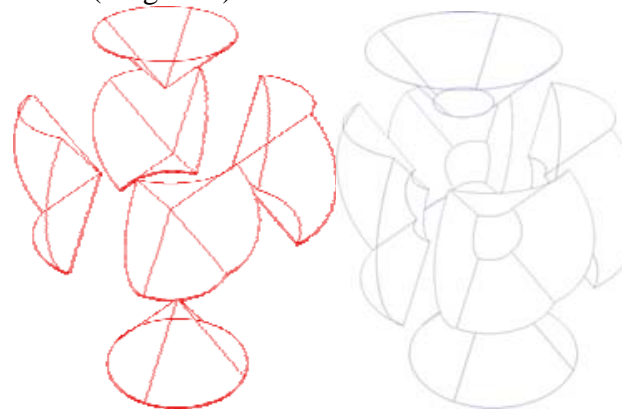

Figure 11. The basic cells of sphere shell volume.

The following operation is the recursive subdivision of each sphere shell volume cell. There are three kinds of subdivision : regular octree subdivision (ROS), degraded octree subdivision (DOS) and adaptive octree subdivision (AOS).

4.4.1 Regular Octree Subdivision: The basic structure of $3 \mathrm{D}$ grid is octree. According to the regular octree structure, one grid cell is subdivided into eight subcells. The regular octree subdivision is took place both at surface and radial. The subdivision of first three subdivision levels is shown in Finger 12 and 13.

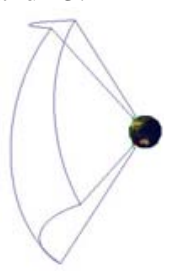

(a) level $=0$

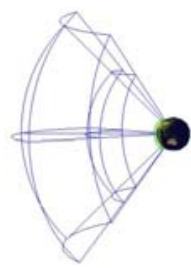

(b) level $=1$

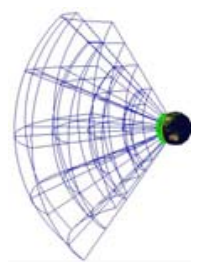

(c) level $=2$
Figure 12. ROS near equator

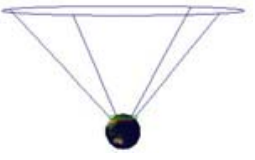

(a) level $=0$

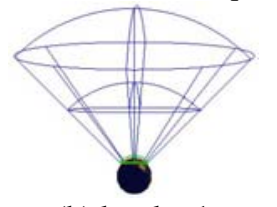

(b) level $=1$

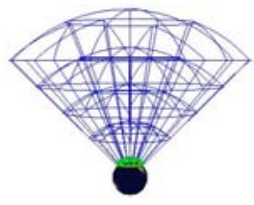

(c) level $=2$
Figure 13. ROS near north polar

4.4.2 Degraded Octree Subdivision: It is obvious that these subcells of ROS are large differences in volume. One feasible way to mitigate the effect is degradation. These four inner subcells are combined into one subcell. The DOS results are shown in Figure 14 and 15. It is noteworthy that outside subdivision is denser than inside.

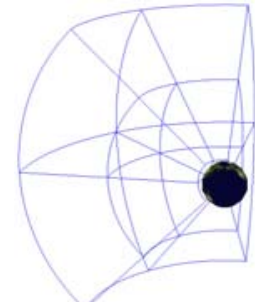

(a) level $=0$

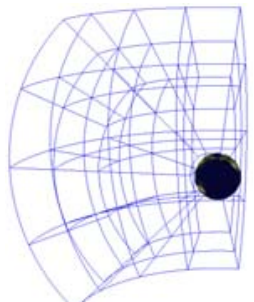

(b) level $=1$

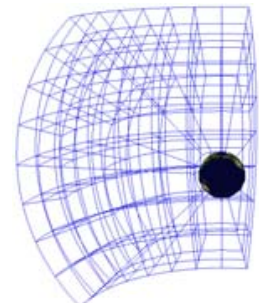

(c) level $=2$
Figure 14. DOS near equator

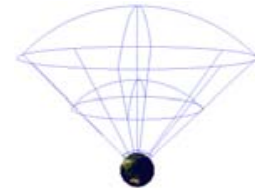

(a) level $=0$

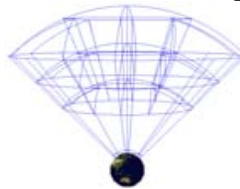

(b) level $=1$

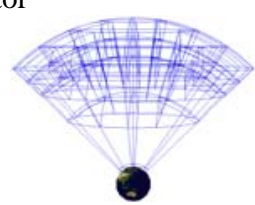

(c) level $=2$
Figure 15. DOS near north polar

4.4.3 Adaptive Octree Subdivision: In fact, the subdivision on surface or radial could be executed several times first, and then the subdivision operation takes place synchronously. In Figure 16, the subdivision is performed on surface independently, while Figure 17 on radial.

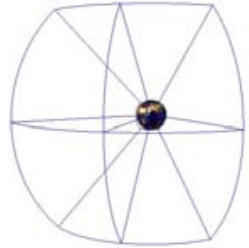

(a) level $=0$

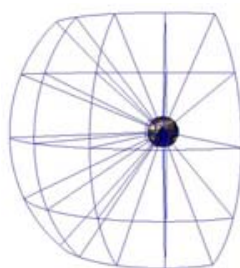

(b) level $=1$

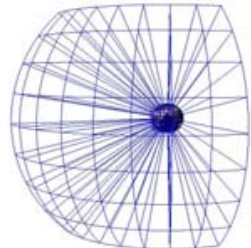

(c) level $=2$
Figure 16. AOS near equator

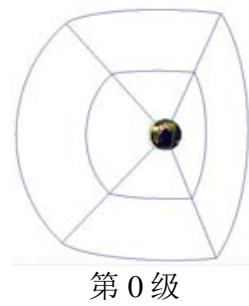

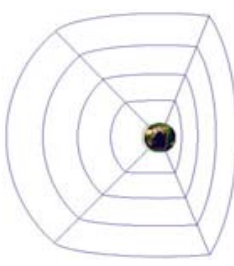

第 1 级

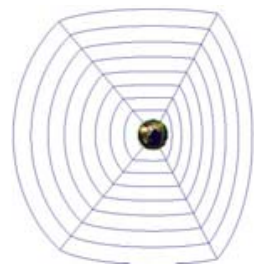

第 2 级
Figure 17. AOS near north polar

\subsection{Coding}

The basic idea of grid code format is $=(l, k, j, i)$, as shown in Figure 18. $l$ denotes the subdivided level, $k, j, i$ denotes the number on each dimensional.

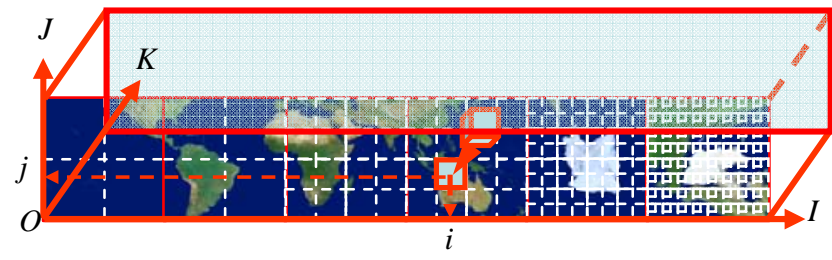

Figure 18. The e-Octree coding structure

Such a basic code $(l, k, j, i)$ could be directly used for ROS. It should be added some parameters for DOS and AOS. For DOS, it should include the degradation flag. For AOS, it should include the independent subdivision level.

The grid code formalization format is

SolidCellCode $=\{$ DatumSphereTag, SolidSubdivideTag $\}$. In this format,

DatumSphereTag $=\{$ DatumSphereID $\mid$ DatumSphereID $\in \mathbf{Z}\}$. 
SolidSubdivideTag $=\{($ RootSolidID, Level, RadialSegID, SphericalRowID, SphericalColID ) | RootSolidID, Level,

RadialSegID, SphericalRowID, SphericalColID $\in \mathbf{N}\}$. DatumSphereTag denotes the tag of datum sphere, DatumSphereID denotes the number of datum sphere, SolidSubdivideTag denotes the subdivision method, RootSolidID denotes the root cell, Level denotes the sundivision level, RadialSegID denotes the number of radial cell segment, SphericalRowID denotes the row number of grid cell on the sphere shell surface, SphericalColID denotes the column number of grid cell on the sphere shell surface.

\subsection{Comparison}

There are points of similarity between Sphere Shell Space 3D Grid (SSSG) and Sphere Degenerated Octree Grid (SDOG). Both of them show homocentric structure, and are based on octree structure. But there are important differences also.

The construction of SDOG is compared to partition a watermelon. The subdivision process is based on degenerated octree structure. In this way, the earth system space is considered as a whole object, so that SDOG has to fix up the radius firstly. Different domains need different radius. As a result, it is hard to integrate multi-domains information.

The construction of SSSG is composed with several sphere shells. The subdivision of different datum sphere shells is separately. Then it is convenient to add another sphere shell or combine two sphere shells, while the subdivision and coding keeps the same.

\subsection{Experiment}

The prototype system of SSSG is realized. And then it has been used to implement global terrain visualization, space orbit objects representation. Both examples run on a laptop with Core2 Duo CPU 2.0GHz, 8600M GT, 1280 800, Microsoft Visual Studio 2005, OpenGL 2.0.

(1) Global terrain visualization

In this example, testing data include SRTM elevation (30 senconds resolution, 3.56GB), BlueMarble Image (30 senconds resolution, 3.6GB) and vector map $(1: 4,000,000$, National Borderline 1.1MB, Province Border-line 1.7MB, Roadway and Railway 17MB). Figure 19 and 20 show Sphere Shell Space 3D Grid over the whole earth and local region.

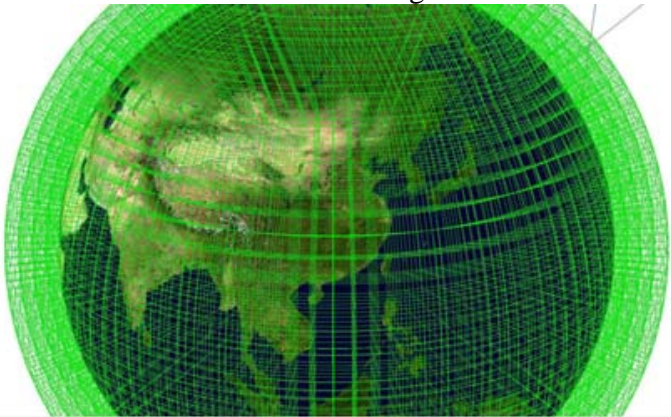

Figure 19. Sphere Shell Space 3D Grid (green wire frame) over the whole earth.

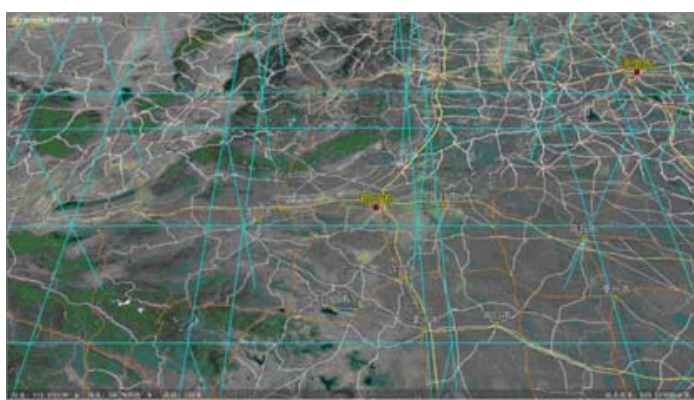

Figure 20. SSSG (blue wire frame) over local region. (2) Space orbit objects representation

In this example, the satellite position is described in SSSG grid code form dynamicly (Figure 21).

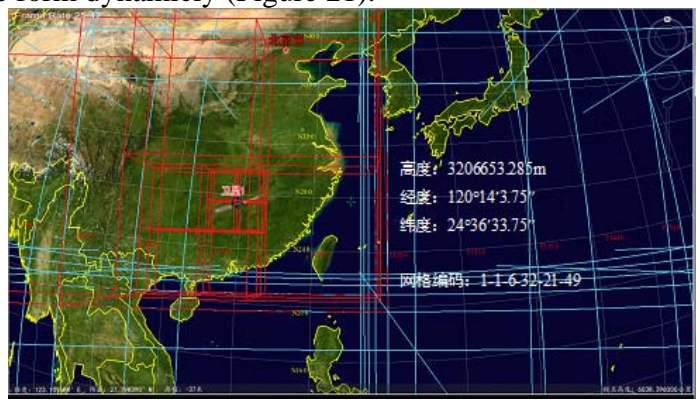

(a)

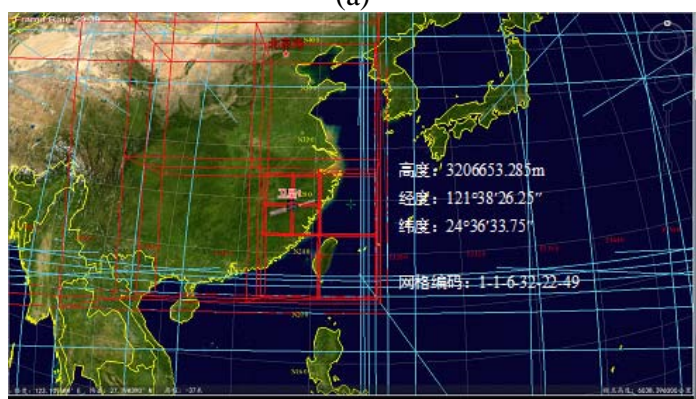

(b)

Figure 21. One moving satellite in the SSSG.

These typical applications experiments show that SSSG is a new 3D spatial reference model for digital earth and a new methodology for global change researches and earth system science.

\section{CONCLUSION}

Sphere Shell Space 3D Grid (SSSG) is proposed. The basic conceptions are introduced, and then the design idea and the compare between SSSG and SDOG are put forward. 10 requirements of SSSG have been presented. Sphere Shell Space 3D Grid is designed to represent spatial objects distributed from underground, land, sea, air to space within a uniform framework, and also to uniformly organize various kinds of spatial information in each earth sphere shells. In theory, SSSG has the ability of represent any space object and process.SSGS provides a new spatial reference framework for the whole earth 3D space for Digital Earth.

\section{REFERENCES}

Cui Majun, Zhao Xuesheng. (2007) Tessellation and distortion analysis based on spherical DQG. Geography and GeoInformation Science, 23(6), pp.23 25.

Dutton, G. (1999) A hierarchical coordinate system for geoprocessing and cartography. Springer-Verlag . Berlin, Germany. 
Fekete, G. (1990) Rendering and managing spherical data with sphere quadtrees. Proceedings of the Visualization'90 IEEE Computer Society, pp.176-186.

Goodchild M. (2000) Discrete global grids for digital earth. In: International Conference on Discrete Global Grids, California: Santa Barbara.

Kageyama A, Tetsuya Sato. (2004) The “Yin-Yang Grid” : An Overset Grid in Spherical Geometry. Geochenmistry Geophisics Geosystems, 5(9), pp.1-15.

Ottosm, Hauska. (2002) Ellipsoidal quadtree for indexing of global geographical data. International Journal of Geographical Information Science, 16(3), pp.213-226.

Sahr K, White D, Kimerling A. (2003) Geodesic Discrete Global Grid Sytems. Cartography and Geographic Information Science, 30(2), pp.121-134.

Seong, J. C. (2005) Implementation of an equal-area girding method for global-scale image archiving. Photogrammetric Engineering \& Remote Sensing, 71(5), pp.623-627.

Stadler G, Gurnis M, Burstedde C. (2010) The Dynamics of Plate Tectonics and Mantle Flow: From Local to Global Scales. Science, 329(5995) , pp.1033-1038.

Tsuboi S, Komatitsch D. JI C. (2008) Computations of global sisimic wave propageation in three dimensional Earth mode. High Performance Computing. pp. 434-443.

Wickman, F. E., Elvers, E., Edvarson, K. (1974) A system of domains for global sampling problems. Geografiska Annaler, 56(3/4), pp.201 212.

YU Jieqing, WU Lixin. (2009) Spatial subdivision and coding of a global three-dimensional grid: spheroid degenerated-octree grid. International geoscience \& remote sensing symposium, Capetown, Africa.

\section{ACKNOWLEDGEMENTS}

This publication is based on work supported by the National Natural Science Foundation of China (Grant No. 41371384). 